PROCEEDINGS OF THE

AMERICAN MATHEMATICAL SOCIETY

Volume 135, Number 9, September 2007, Pages 2831-2838

S 0002-9939(07)08910-1

Article electronically published on February 9, 2007

\title{
STRONG CONVERGENCE OF PATH FOR CONTINUOUS PSEUDO-CONTRACTIVE MAPPINGS
}

\author{
CLAUDIO H. MORALES
}

(Communicated by Joseph A. Ball)

\begin{abstract}
The purpose of this paper is to study the convergence of a path that begins at the unique fixed point of a strongly pseudo-contractive operator defined on a closed and convex subset of a reflexive Banach space and converges to a fixed point of a pseudo-contractive mapping. Primarily, it is proven that a convex combination of these two operators is indeed strongly pseudo-contractive under the weakly inward condition. This fact generalizes a result of Barbu for accretive operators.
\end{abstract}

\section{INTRODUCTION}

Let $X$ be a (real) Banach space. An operator $T: D(T) \subset X \rightarrow 2^{X}$ is said to be $k$-pseudo-contractive $(k \in \mathbb{R})$ if for each $u, v \in D(A)$ there exists $j \in J(u-v)$ such that

$$
\langle u-v, j\rangle \leq k\|x-y\|^{2}
$$

for all $u \in T x$ and $v \in T y$, where $J: X \rightarrow X^{*}$ is the normalized duality mapping which is defined by

$$
J(u)=\left\{j \in X^{*}:\langle u, j\rangle=\|u\|^{2},\|j\|=\|u\|\right\} .
$$

Here $\langle.,$.$\rangle denotes the generalized duality pairing. For 0<k<1$ in the inequality (1), we say that $T$ is strongly pseudo-contractive, while for $k=1, T$ is simply called pseudo-contractive. It is worthy to mention that this latter class of operators was first introduced by Browder [5. It is an immediate consequence of the HahnBanach Theorem that $J(u)$ is nonempty for each $u$ in $X$. It is also known that $J(u)$ is single-valued if and only if $X$ is smooth. By the way, this latter notation means that

$$
\lim _{t \rightarrow 0} \frac{\|x+t h\|-\|x\|}{t}
$$

exists for each $x$ and $h$ on the unit sphere $U$ of $X$. When this is the case, we say that the norm of $X$ is Gâteaux differentiable. Moreover, if for each $h$ in $U$ the limit defined by (2) is uniformly attained for $x$ in $U$, we say that the norm of $X$ is uniformly Gâteaux differentiable. It is also known that if $X$ has a uniformly Gâteaux

Received by the editors May 23, 2006.

2000 Mathematics Subject Classification. Primary 47H10; Secondary 65J15.

Key words and phrases. Pseudo-contractive operators, weakly inward condition, reflexive Banach spaces, uniformly Gâtaux differentiable norm. 
differentiable norm, then the mapping $J$ is uniformly continuous on bounded subsets of $E$ from the strong topology on $E$ to the weak* topology on $E^{*}$.

In addition to generalizing the nonexpansive mappings (mappings $T: D \rightarrow X$ for which $\|T x-T y\| \leq\|x-y\|$ for all $x$ and $y$ in $D$ ), the pseudo-contractive ones are characterized by the fact that $T$ is pseudo-contractive if and only if $I-$ $T$ is accretive (see [4, 10]). Continuous interest in mapping theory for accretive operators, particularly as it relates to existence theorems for nonlinear ordinary and partial differential equations, has prompted a corresponding interest in fixed point theory for pseudo-contractive mappings (see, e.g., [5], 8], 9], 10, 11, 14, [16]).

It is relevant to the first two theorems of this paper to note that while every uniformly smooth Banach space is a reflexive Banach space with a uniformly Gâteaux differentiable norm, the converse does not hold. To see this, consider $X$ to be the direct sum $l^{2}\left(l^{p_{n}}\right)$, the class of all those sequences $x=\left\{x_{n}\right\}$ with $x_{n} \in l^{p_{n}}$ and $\|x\|=\left(\sum_{n<\infty}\left\|x_{n}\right\|^{2}\right)^{\frac{1}{2}}$ (see [15]). Now, if $1<p_{n}<\infty$ for all $n \in \mathbb{N}$, where either $\lim \sup _{n \rightarrow \infty} p_{n}=\infty$ or $\liminf _{n \rightarrow \infty} p_{n}=1$, then $X$ is a reflexive Banach space (see [15. again)with a uniformly Gâteaux differentiable norm (see [21]), but is not uniformly smooth (see [15]). We also observe that spaces which enjoy the fixed point property (F.P.P.) for nonexpansive self-mappings are not necessarily spaces with a uniformly Gâteaux differentiable norm. On the other hand, the converse of this fact appears to be unknown as well.

The purpose of this paper is twofold. First of all, we prove that continuous accretive operators defined on convex domains under the assumption that $I-A$ satisfies the weakly inward condition, hold the duality formulation of accretiveness for all functionals $j$ in the corresponding duality mapping. This can be written as follows:

$$
\langle A(x)-A(y), j\rangle \geq 0 \text { for all } j \in J(x-y) .
$$

This result is, in fact, an extension of one obtained by Barbu [2]. Secondly, as a consequence of this, we obtain a quite general result for the existence of a path that begins at the unique fixed point of one operator and ends at a fixed point of the second operator, which appears to be the unique solution of a variational inequality. This latter result extends recent works of Moudafi [18, $\mathrm{Xu}$ [20] and Chen et al [7, as well as the main theorem of [17].

Throughout the paper we will use the following notion. Let $X$ be a linear space with $K \subset X$. For $x \in K$, the inward set $I_{K}(x)$ is defined by

$$
I_{K}(x)=\{x+\lambda(u-x): u \in K, \lambda \geq 0\} .
$$

A mapping $T: K \rightarrow X$ is said to satisfy the weakly inward condition if

$$
T x \in \overline{I_{K}(x)} \text { for all } x \in K \text {. }
$$

On the other hand, if $T x \in I_{K}(x)$ for each $x \in K, T$ is said to, simply, satisfy the inward condition. We denote by Fix $(T)$ the set of fixed points of $T$.

\section{Global PROPERTy}

We begin with a theorem that extends a result of Barbu [2]. The argument of the proof was inspired by a tool used by Kirk and Schöneberg [12. To accomplish this we need the following lemma due to Kato [10]. 
Lemma 1. Let $X$ be a (real) Banach space. Then $\|x\| \leq\|x+\alpha y\|$ for all $\alpha>0$ if and only if there exists $j \in J(x)$ such that $\langle y, j\rangle \geq 0$.

As a consequence of Lemma 1 we derive a well-known characterization of accretiveness.

Lemma 2. Let $X$ be a (real) Banach space. Then $A: D(A) \rightarrow X$ is accretive if and only if

$$
\|x-y\| \leq\|x-y+\alpha(A(x)-A(y))\| \text { for all } x, y \in D(A) \text { and } \alpha>0 .
$$

Theorem 1. Let $K$ be a convex subset of a Banach space $X$. Suppose $A$ is a continuous and accretive mapping from $K$ into $X$ such that $I-A$ enjoys the weakly inward condition. Let $x, y \in K$. Then

$$
\langle A(x)-A(y), j\rangle \geq 0 \text { for all } j \in J(x-y) .
$$

Proof. Let $x, y \in K$ and define $\gamma:[0,1] \times[0,1] \rightarrow X$ by $\gamma(t, s)=z_{t}-s A\left(z_{t}\right)$, where $z_{t}=t x+(1-t) y$. Let $j \in J(x-y)$, and define the set $M$ by

$$
M=\left\{t \in[0,1]:\left\langle A\left(z_{t}\right)-A(y), j\right\rangle \geq 0\right\} .
$$

Then $M$ is nonempty $(0 \in M)$ and continuity of $A$ implies that $t_{0}=\sup (M) \in M$. Since $z_{t}-A\left(z_{t}\right) \in \overline{I_{K}\left(z_{t}\right)}$ for $t \in[0,1]$, then for a given $\epsilon>0$, there exists $v_{t} \in I_{K}\left(z_{t}\right)$ such that $\left\|z_{t}-A\left(z_{t}\right)-v_{t}\right\|<\epsilon$. This implies

$$
v_{t}=\left(1-\lambda_{t}\right) z_{t}+\lambda_{t} u_{t} \text { for some } u_{t} \in K \text { and } \lambda_{t} \geq 1 \text {. }
$$

Define $\delta(t, s)=\left(1-s \lambda_{t}\right) z_{t}+s \lambda_{t} u_{t}$ for $s \in[0,1]$. This means that $\gamma(t, s)=$ $\delta(t, s)+s e_{t}$, where $e_{t}=z_{t}-A\left(z_{t}\right)-v_{t}$ and $\left\|e_{t}\right\|<\epsilon$. Select $t \in\left(t_{0}, 1\right)$. Then there exists $s_{0} \in(0,1)$ such that $\delta(t, s)$ and $\delta\left(t_{0}, s\right)$ are in $K$ for all $s \in\left(0, s_{0}\right]$. Consequently, by Lemma 2, we have

$$
\begin{aligned}
\left\|\gamma(t, s)-\gamma\left(t_{0}, s\right)\right\| \leq & \left\|\delta(t, s)-\delta\left(t_{0}, s\right)\right\|+s\left\|e_{t}-e_{t_{0}}\right\| \\
\leq & \| \delta(t, s)-\delta\left(t_{0}, s\right)+s\left(A(\delta(t, s))-A\left(\delta\left(t_{0}, s\right)\right)\|+s\| e_{t}-e_{t_{0}} \|\right. \\
\leq & \left\|z_{t}-z_{t_{0}}\right\|+s\left\|A(\delta(t, s))-A\left(z_{t}\right)\right\|+s\left\|A\left(\delta\left(t_{0}, s\right)\right)-A\left(z_{t_{0}}\right)\right\| \\
& +2 s\left\|e_{t}-e_{t_{0}}\right\|
\end{aligned}
$$

for all $s \in\left(0, s_{0}\right]$. This implies that

$$
\begin{aligned}
\left\langle A\left(z_{t}\right)-A(y), j\right\rangle= & \left\langle A\left(z_{t}\right)-A\left(z_{t_{0}}\right), j\right\rangle+\left\langle A\left(z_{t_{0}}\right)-A(y), j\right\rangle \\
\geq & \left\langle A\left(z_{t}\right)-A\left(z_{t_{0}}\right), j\right\rangle \\
= & \frac{1}{s}\left[\left\langle z_{t}-z_{t_{0}}, j\right\rangle-\left\langle\gamma(t, s)-\gamma\left(t_{0}, s\right), j\right\rangle\right] \\
\geq & \frac{1}{s}\|x-y\|\left[\left\|z_{t}-z_{t_{0}}\right\|-\left\|\gamma(t, s)-\gamma\left(t_{0}, s\right)\right\|\right] \\
\geq & -\|x-y\|\left[\left\|A(\delta(t, s))-A\left(z_{t}\right)\right\|+\left\|A\left(\delta\left(t_{0}, s\right)\right)-A\left(z_{t_{0}}\right)\right\|\right. \\
& \left.+2\left\|e_{t}-e_{t_{0}}\right\|\right],
\end{aligned}
$$

so by letting $s \rightarrow 0^{+}$, we obtain that $\left\langle A\left(z_{t}\right)-A(y), j\right\rangle \geq-4 \epsilon\|x-y\|$. Since $\epsilon$ is an arbitrary positive number, $\left\langle A\left(z_{t}\right)-A(y), j\right\rangle \geq 0$, and hence $t \in M$, which is a contradiction! Therefore, $t_{0}=1$, and hence $\langle A(x)-A(y), j\rangle \geq 0$. Since $j \in J(x-y)$ was arbitrary, the proof is complete. 
We remark that if we assume that the domain of the operator $A$ is open and convex, the assumption that $I-A$ is weakly inward can be dropped, as may be seen in the following corollary.

Corollary 1. Let $D$ be an open and convex subset of a Banach space X. Suppose $A$ is a continuous and accretive mapping from $D$ into $X$. Then for every $x, y \in D$,

$$
\langle A(x)-A(y), j\rangle \geq 0 \text { for all } j \in J(x-y) .
$$

Proof. As in the proof of Theorem 1, we know that $z_{t_{0}} \in D$ and since $D$ is open, there exists an open ball $B\left(z_{t_{0}} ; r\right) \subset D$ for some $r>0$. Since the mapping $\gamma$ is continuous at $\left(t_{0}, 0\right)$, there exists $t \in\left(t_{0}, 1\right)$ and $s_{0} \in(0,1)$ such that $\gamma(t, s) \in$ $B\left(z_{t_{0}} ; r\right)$ for all $s \in\left(0, s_{0}\right]$. Since $A$ is accretive we obtain $\left\|\gamma(t, s)-\gamma\left(t_{0}, s\right)\right\| \leq\left\|z_{t}-z_{t_{0}}\right\|+s\left\|A(\gamma(t, s))-A\left(z_{t}\right)\right\|+s\left\|A\left(\gamma\left(t_{0}, s\right)\right)-A\left(z_{t_{0}}\right)\right\|$ for all $s \in\left(0, s_{0}\right]$. The remainder part of the proof is as in the proof of Theorem 1 .

For general Banach spaces, it appears to be unknown whether a convex combination of two pseudo-contractive mappings is pseudo-contractive. The next corollary gives an answer to this question.

Corollary 2. Let $K$ be a closed convex subset of a Banach space $X$. Suppose $T, S$ are continuous and pseudo-contractive mappings from $K$ into $X$ such that both enjoy the weakly inward condition. Then $h=t T+(1-t) S$ is pseudo-contractive for each $t \in[0,1]$ that also enjoys the weakly inward condition. In addition, for $x, y \in K$,

$$
\langle h(x)-h(y), j\rangle \leq\|x-y\|^{2} \text { for all } j \in J(x-y) .
$$

Proof. By Theorem 1, $I-T$ and $I-S$ are both accretive, where (3) holds for all $j \in J(x-y)$. Consequently, $I-h$ is accretive while $h$ satisfies the weakly inward condition. Hence $I-h$ satisfies (3), which completes the proof.

Corollary 3. Let $X$ be a Banach space, and let $A: D(A) \subset X \rightarrow X$ be an maccretive operator while $B: \overline{c o}(D(A)) \rightarrow X$ is continuous and accretive where $I-B$ enjoys the weakly inward condition. Then $A+B$ is m-accretive.

Proof. Since $B$ satisfies all the assumptions of Theorem 1,

$$
\langle B(x)-B(y), j\rangle \geq 0 \text { for all } j \in J(x-y) .
$$

Hence $A+B$ is accretive. Now, due to Theorem 5.3 of [13], we conclude that $A+B$ is $m$-accretive.

We remark that Corollary 3 extends Theorem 1 of Barbu [1]. We may also observe that, in the context of Corollary 2, the sum of two pseudo-contractive mappings is also pseudo-contractive, provided that one of the two is continuous and satisfies the weakly inward condition.

\section{CONVERGENCE OF PATH}

Recently, the use of path convergency has awakened interest in proving the convergence of iterative methods to approximate fixed points. For instance, see Xu [19] and Chidume and Zegeye [6. Following this direction, we first prove the existence of a path for a convex combination of a pseudo-contractive type of operators. 
Proposition 1. Let $K$ be a closed convex subset of a Banach space $X$. Suppose $T, h$ are two continuous mappings from $K$ into $X$, which are pseudo-contractive and strongly pseudo-contractive, respectively. Suppose, in addition, $T$ and $h$ satisfy the weakly inward condition. Then there exists a unique path $t \mapsto x_{t} \in K, t \in[0,1)$, satisfying

$$
x_{t}=t T\left(x_{t}\right)+(1-t) h\left(x_{t}\right) .
$$

Proof. For each $t \in[0,1)$, the mapping $T_{t}(x)=t T(x)+(1-t) h(x)$ is continuous, and due to Theorem 1, it is also strongly pseudo-contractive, satisfying the weakly inward condition. Therefore, $T_{t}$ has a unique fixed point in $K$. This means that the equation

$$
x_{t}=t T\left(x_{t}\right)+(1-t) h\left(x_{t}\right)
$$

has a unique solution for each $t \in[0,1)$. To see the continuity, let $t, t_{0} \in[0,1)$. Then there exists $j \in J\left(x_{t}-x_{t_{0}}\right)$ such that

$$
\begin{aligned}
\left\langle x_{t}-x_{t_{0}}, j\right\rangle= & t\left\langle T\left(x_{t}\right)-T\left(x_{t_{0}}\right), j\right\rangle+(1-t)\left\langle h\left(x_{t}\right)-h\left(x_{t_{0}}\right), j\right\rangle \\
& +\left(t-t_{0}\right)\left\langle T\left(x_{t_{0}}\right)-h\left(x_{t_{0}}\right), j\right\rangle,
\end{aligned}
$$

and this implies that

$\left\|x_{t}-x_{t_{0}}\right\|^{2} \leq[t+(1-t) k]|| x_{t}-x_{t_{0}}\left\|^{2}+\left|t-t_{0}\right|\right\| T\left(x_{t_{0}}\right)-h\left(x_{t_{0}}\right)\|\| x_{t}-x_{t_{0}}||$.

Therefore,

$$
\left\|x_{t}-x_{t_{0}}\right\| \leq \frac{\| T\left(x_{t_{0}}\right)-h\left(x_{t_{0}}\right)||}{(1-t)(1-k)}\left|t-t_{0}\right|,
$$

which completes the proof.

Proposition 2. Let $K$ be a closed convex subset of a Banach space $X$ and let $T$ be pseudo-contractive, while $h$ is strongly pseudo-contractive on K. Suppose either $T$ or $h$ enjoys the weakly inward condition, and suppose that the equation

$$
x=t T(x)+(1-t) h(x)
$$

has a solution $x_{t}$ for each $t \in(0,1)$. Then:

(i) Suppose there exists a bounded sequence $\left\{x_{n}\right\}$ in $K$ such that $x_{n}-T\left(x_{n}\right) \rightarrow 0$, while $\left\{x_{n}-h\left(x_{n}\right)\right\}$ is bounded. Then the path $\left\{x_{t}\right\}$ is bounded.

(ii) In particular, if $T$ has a fixed point in $K$, then the path $\left\{x_{t}\right\}$ is also bounded.

(iii) If $v$ is a fixed point of $T$, there exists $j \in J\left(x_{t}-v\right)$ such that

$$
\left\langle x_{t}-h\left(x_{t}\right), j\right\rangle \leq 0 \text {. }
$$

Proof. (i) Since $T$ and $h$ are pseudo-contractive and strongly pseudo-contractive, respectively, we may choose $j \in J\left(x_{t}-x_{n}\right)$ such that

$$
\begin{aligned}
\left\langle x_{t}-x_{n}, j\right\rangle= & t\left\langle T\left(x_{t}\right)-T\left(x_{n}\right), j\right\rangle+(1-t)\left\langle h\left(x_{t}\right)-h\left(x_{n}\right), j\right\rangle \\
& +t\left\langle T\left(x_{n}\right)-x_{n}, j\right\rangle+(1-t)\left\langle h\left(x_{n}\right)-x_{n}, j\right\rangle .
\end{aligned}
$$

This implies that

$$
(1-t)(1-k)\left\|x_{t}-x_{n}\right\| \leq t\left\|x_{n}-T\left(x_{n}\right)\right\|+(1-t)\left\|x_{n}-h\left(x_{n}\right)\right\|,
$$

and thus

$$
\limsup _{n \rightarrow \infty}\left\|x_{t}-x_{n}\right\| \leq \frac{1}{1-k} \limsup _{n \rightarrow \infty}\left\|x_{n}-h\left(x_{n}\right)\right\| .
$$

Therefore the path $\left\{x_{t}\right\}$ is bounded. 
(ii) The proof of (ii) is a direct consequence of part (i).

(iii) Let $v$ be a fixed point of $T$. By the pseudo-contractiveness, there exists $j \in J\left(x_{t}-v\right)$ such that

$$
\begin{aligned}
\left\langle x_{t}-h\left(x_{t}\right), j\right\rangle & =\frac{t}{1-t}\left\langle T\left(x_{t}\right)-x_{t}, j\right\rangle \\
& =\frac{t}{1-t}\left[\left\langle T\left(x_{t}\right)-T(v), j\right\rangle+\left\langle v-x_{t}, j\right\rangle\right] \\
& \leq \frac{t}{1-t}\left[\left\langle x_{t}-v, j\right\rangle+\left\langle v-x_{t}, j\right\rangle\right] \leq 0 .
\end{aligned}
$$

Theorem 2. Let $K$ be a closed convex subset of a reflexive Banach space $X$ with a uniformly Gâteaux differentiable norm. Let $T, h$ be pseudo-contractive and strongly pseudo-contractive continuous mappings from $K$ into $X$, respectively. Suppose $T$ and $h$ satisfy the weakly inward condition and every closed, bounded and convex subset of $K$ has the F.P.P. for nonexpansive self-mappings. If the set

$$
E=\{x \in K: T(x)=\lambda x+(1-\lambda) h(x) \text { for some } \lambda>1\}
$$

and $h(E)$ are bounded, then the path $\left\{x_{t}: t \in[0,1)\right\}$ described by (4) converges strongly to a fixed point $v$ of $T$ as $t \rightarrow 1^{-}$. In addition, this fixed point is the unique solution of the variational inequality:

$$
\langle v-h(v), J(v-w)\rangle \leq 0 \text { for all } w \in \operatorname{Fix}(T) .
$$

Proof. By Proposition 1 the path $\left\{x_{t}: t \in[0,1)\right\}$ exists; it remains to show that it converges strongly to a fixed point of $T$ as $t \rightarrow 1^{-}$. The mapping $2 I-T$ has a nonexpansive inverse, denoted by $g$, which maps $K$ into $K$ as a consequence of Theorem 6 of [14. Let $x_{n}=x_{t_{n}}$ where $\left\{t_{n}\right\}$ is a sequence in $[0,1)$ with $t_{n} \rightarrow 1^{-}$as $n \rightarrow \infty$. Since

$$
x_{n}-T\left(x_{n}\right)=\left(1-t_{n}^{-1}\right)\left(x_{n}-h\left(x_{n}\right)\right),
$$

and the sets $E$ and $h(E)$ are bounded, we derive that $x_{n}-T\left(x_{n}\right) \rightarrow 0$ as $n \rightarrow \infty$. This implies that $x_{n}-g\left(x_{n}\right) \rightarrow 0$. Since $\left\{x_{n}\right\}$ is bounded, we may define $f: X \rightarrow$ $\mathbb{R}^{+}$by $f(x)=\limsup \left\|x_{n}-x\right\|$. Then $f$ is continuous and convex, and therefore the set

$$
K_{0}=\{u \in K: f(u)=\inf \{f(x): x \in K\}\}
$$

is a nonempty (due to Theorem 1.2 of $[3$ ), closed, bounded and convex subset of $K$. Since $f(g(u)) \leq f(u)$ for all $u \in K_{0}, K_{0}$ is also invariant under $g$. Consequently, due to the assumption on $K, g$ has a fixed point in $K_{0}$. Denote such a point by $v$. Since $T$ is pseudo-contractive and $v$ is a fixed point of $T$, we derive from Proposition 2(iii) that

$$
\left\langle x_{n}-h\left(x_{n}\right), J\left(x_{n}-v\right)\right\rangle \leq 0
$$

for all $n \in \mathbb{N}$. Now let $x=y-v$, with $y \in I_{K}(v)$ and $t \in(0,1]$. Then by a well-known inequality (see, for instance, Lemma 1 of [17]) we have

$$
\left\|x_{n}-v-t x\right\|^{2} \leq\left\|x_{n}-v\right\|^{2}+2\left\langle-t x, J\left(x_{n}-v-t x\right)\right\rangle .
$$

Let $\varepsilon>0$. Then by the assumptions on $X$ and the fact that $y \in I_{K}(v)$, there exists $\delta>0$ such that $v+t x \in K$ and

$$
\left\langle x, J\left(x_{n}-v\right)\right\rangle<\varepsilon+\left\langle x, J\left(x_{n}-v-t x\right)\right\rangle
$$


for all $t \in(0, \delta)$. Consequently,

$$
\left\langle x, J\left(x_{n}-v\right)\right\rangle<\varepsilon+\frac{1}{2 t}\left[\left\|x_{n}-v\right\|^{2}-\left\|x_{n}-v-t x\right\|^{2}\right] .
$$

Since $h(v) \in \overline{I_{K}}(v)$, we may choose $y \in I_{K}(v)$ as close as is needed from $h(v)$, and we may choose $\delta_{y}>0$ such that $(1-t) v+t y \in K$ for all $t \in\left(0, \delta_{y}\right)$ so that

$$
\left\langle y-v, J\left(x_{n}-v\right)\right\rangle<\varepsilon+\frac{1}{2 t}\left[\left\|x_{n}-v\right\|^{2}-\left\|x_{n}-[(1-t) v+t y]\right\|^{2}\right] .
$$

Therefore we may choose a subsequence $\left\{x_{n_{k}}\right\}$ of $\left\{x_{n}\right\}$ such that

$$
\limsup _{k \rightarrow \infty}\left\langle h(v)-v, J\left(x_{n_{k}}-v\right)\right\rangle \leq 0 .
$$

Combining (5) and (6) and the fact that $h$ is strongly pseudo-contractive, we conclude that $\left\{x_{n_{k}}\right\}$ converges strongly to $v$. To prove that actually the net $\left\{x_{t}\right\}$ converges to $v$, let $\left\{x_{m_{k}}\right\}$ be another subsequence of $\left\{x_{t}: t \in[0,1)\right\}$ such that $x_{m_{k}}=x_{t_{m_{k}}}, t_{m_{k}} \rightarrow 1^{-}$as $k \rightarrow \infty$, and $x_{m_{k}} \rightarrow w$, where $T w=w$. Then Proposition 2(iii) implies that $\langle v-h(v), J(v-w)\rangle \leq 0$ and $\langle w-h(w), J(w-v)\rangle \leq 0$. This implies that

$$
\langle v-w-(h(v)-h(w)), J(v-w)\rangle \leq 0 .
$$

Since $h$ is strongly pseudo-contractive, $v=w$ and the strong $\lim _{t \rightarrow 1^{-}} x_{t}$ exists. This very same argument may be used to conclude that $v$ is the only solution of the variational inequality

$$
\langle v-h(v), J(v-w)\rangle \leq 0 \text { for all } w \in F i x(T) .
$$

We remark that Theorem 2 extends Theorem 2.1 of [18], Theorem 4.1 of [19] and Theorem 1 of [17]. Also, we derive two corollaries, which are extensions of Theorem 3 of [7] on various aspects. Perhaps, the most interesting aspect of this result is its generality, which includes the contractions as the initial mapping $h$. In fact, throughout the mapping $F(t, x)=t T(x)+(1-t) h(x), T$ and $h$ are homotopically equivalent under suitable boundedness conditions. We observe an identification between the unique fixed point of a contraction and a fixed point of a pseudocontraction.

Corollary 4. Let $K$ be a closed convex subset of a uniformly smooth Banach space $X$. Let $T, h$ be pseudo-contractive and strongly pseudo-contractive continuous mappings from $K$ into $X$, respectively. Suppose $T$ and $h$ satisfy the weakly inward condition. If the set

$$
E=\{x \in K: T(x)=\lambda x+(1-\lambda) h(x) \text { for some } \lambda>1\}
$$

and $h(E)$ are bounded, then the path $\left\{x_{t}: t \in[0,1)\right\}$ described by (4) converges strongly to a fixed point of $T$ as $t \rightarrow 1^{-}$.

Corollary 5. Let $K$ be a closed convex subset of a uniformly smooth Banach space $X$. Let $T, h$ be pseudo-contractive and strongly pseudo-contractive continuous mappings from $K$ into $X$, respectively. Suppose $T$ and $h$ satisfy the weakly inward condition. If $h$ is bounded and $T$ has a fixed point, then the path $\left\{x_{t}: t \in[0,1)\right\}$ described by (4) converges strongly to a fixed point of $T$ as $t \rightarrow 1^{-}$. 
Proof. Since $T$ has a fixed point, then by Proposition $2(\mathrm{i})$, the path $\left\{x_{t}\right\}$ is bounded, and consequently, the set $E$ defined in Theorem 1 is bounded. Also since $h$ is a bounded mapping, $h(E)$ is bounded. Now, the conclusion follows from Theorem 1 .

\section{REFERENCES}

[1] V. Barbu, "Continuous perturbations of nonlinear $m$-accretive operators in Banach spaces", Bollettino U. M. I. 6 (1972), 270-278. MR0326514(48:4858)

[2] V. Barbu, "Nonlinear semigroups and differential equations in Banach spaces", Editura Academiei R.S.R, Bucharest, 1976. MR0390843 (52:11666)

[3] V. Barbu and Th. Preocupanu, "Convexity and optimization in Banach spaces", Editura Academiei R.S.R, Bucharest, 1978. MR.0513634 (58:23939)

[4] F. F. Browder, "Nonlinear operators and nonlinear equations of evolution in Banach spaces", Proc. Symp. Pure Math. 18 (1976), part 2. Amer. Math. Soc., Providence, R.I. MR0405188 $(53: 8982)$

[5] F. E. Browder, "Nonlinear mappings of non-expansive and accretive type in Banach spaces", Bull. Amer. Math. Soc. 73 (1967), 875-882. MR0232255 (38:581)

[6] C. E. Chidume and H. Zegeye, "Approximate fixed point sequences and convergence theorems for Lipschitz pseudocontractive maps", Proc. Amer. Math. Soc. 132 (2004), 831-840. MR2019962 (2004j:47114)

[7] R. Chen, P. K. Lin, and Y. Song, "An approximation method for strictly pseudocontractive mappings", Nonlinear Anal., 64 (2006), 2527-2535. MR2215825 (2006k:47114)

[8] M. G. Crandall, "Differential equations on convex sets", J. Math. Soc. Japan, 22 (1970), 443-455. MR0268491 (42:3388)

[9] K. Deimling "Zeros for accretive operators", Manuscripta Math. 13 (1974), 365-374. MR0350538 (50:3030)

[10] T. Kato, "Nonlinear semigroups and evolution equations", J. Math. Soc. Japan 19 (1967), 508-520. MR0226230 (37:1820)

[11] W. A. Kirk "A fixed point theorem for local pseudo-contractions in uniformly convex Banach spaces", Manuscripta Math., 30 (1979), 98-102. MR0552364 (80k:47064)

[12] W. A. Kirk and R. Schöneberg, "Mapping theorems for local expansions in metric and Banach spaces", J. Math. Anal. Appl. 72 (1979), 114-121. MR0552327 (81c:47053)

[13] Y. Kobayashi, "Difference approximation of Cauchy problems for quasi-dissipative operators and generation of nonlinear semigroups", J. Math. Soc. Japan 27 (1975), 640-665. MR0399974 (53:3812)

[14] R. H. Martin, "Differential equations on closed subsets of a Banach space", Trans. Amer. Math. Soc. 179 (1973), 399-414. MR0318991(47:7537)

[15] M. M. Day, "Reflexive Banach spaces not isomorphic to uniformly convex spaces", Bull. Amer. Math. Soc., 47 (1941), 313-317. MR0003446 (2:221b)

[16] C. H. Morales, "On the fixed-point theory for local $k$-pseudocontractions", Proc. Amer. Math. Soc. 81 (1981), 71-74. MR0589138 (82c:47072)

[17] C. H. Morales and Jong S. Jung, "Convergence of paths for pseudo-contractive mappings in Banach spaces", Proc. Amer. Math. Soc. 128 (2000), 3411-3419. MR1707528 (2001b:47090)

[18] A. Moudafi, "Viscosity approximation methods for fixed-points problems", J. Math. Anal. Appl. 241 (2000), 46-55. MR.1738332 (2000k:47085)

[19] H. K. Xu, "Iterative algorithms for nonlinear operators", J. London Math. Soc. 66 (2002), 240-256. MR1911872 (2003e:47114)

[20] H. K. Xu, "Viscosity approximation methods for nonexpansive mappings", J. Math. Anal. Appl. 298 (2004), 279-297. MR2086546(2005e:47133)

[21] V. Zizler, "On some rotundity and smoothness properties of Banach spaces", Dissert. Math. 87 (1971), 5-33. MR0300060(45:9108)

Department of Mathematics, University of Alabama in Huntsville, Huntsville, Alabama 35899

E-mail address: morales@math.uah.edu 